\title{
Subacute Bilateral Coronary Ostial Stenoses Following Cardiac Catheterization and PTCA
}

\author{
Vance E. Wilson, MD, and Eric R. Bates, MD \\ Accelerated coronary ostial stenosis is an infrequent late complication of coronary artery \\ catheter instrumentation during aortic valve surgery, PTCA, or coronary angiography. We \\ report the first case of bilateral coronary artery ostial stenoses developing after cardiac \\ catheterization and right coronary artery PTCA. In patients with worsening angina fol- \\ lowing cardiac catheterization or PTCA, accelerated ostial stenoses should not be over- \\ looked in the differential diagnosis.
}

Key words: coronary angiography, angina

\section{INTRODUCTION}

Accelerated coronary ostial stenosis following cardiac catheterization or PTCA is fortunately an uncommon complication. We report the first case of bilateral coronary ostial stenoses developing after cardiac catheterization and right coronary artery PTCA.

\section{CASE REPORT}

J.C., a 55-yr-old man, developed atypical right-sided chest pain playing softball in June 1989. Because of a smoking habit and a family history of sudden death, an exercise tolerance test was performed. The patient developed 2-3 $\mathrm{mm}$ of horizontal ST-segment depression in Stage 3 of a Bruce protocol without symptoms.

A cardiac catheterization was performed 1 month later using an 8 French Sones catheter. Coronary angiography revealed intimal irregularity of the proximal circumflex artery and a focal $30 \%$ stenosis of the posterior descending artery. The right coronary ostium was $10 \%$ narrowed and the left coronary ostium was $30 \%$ narrowed (Fig. $1 \mathrm{~A}, \mathrm{C})$. The left ventricular function and remaining coronary artery segments were normal. The stress test was assumed to be false-positive and once-daily aspirin was instituted.

Starting in August 1989, the patient developed typical Class II exertional angina. An exercise test was again positive, but this time with chest pain. On thallium scintigraphy, a large reversible inferolateral defect was noted. In October 1989, the patient underwent PTCA of a $70 \%$ posterior descending artery stenosis with a reduction of stenosis severity to $10 \%$. An 8 French Judkins guiding catheter was used without damping. The left coronary artery was not injected at that time.

Despite successful PTCA, the patient continued to ex- perience exertional angina. A repeat stress thallium study in November 1989 demonstrated ST-segment depression and chest pain in Stage 2. Thallium images revealed only a small fixed posterolateral defect.

Because of worsening angina, the patient was referred to the University of Michigan. Cardiac catheterization was performed in March 1990 using 6 French Judkins catheters. Coronary angiography revealed a $75 \%$ left coronary ostial stenosis and a 60\% right coronary ostial stenosis without reflux of contrast (Fig. 1B,D). Because of pressure-damping, sublingual and intracoronary nitroglycerin were administered without effect. While the site of prior PTCA was widely patent, the left circumflex coronary artery was occluded at the ostium with trace left-to-left collaterals. Immediately after cardiac catheterization, the patient developed rest angina with STsegment changes and underwent urgent 3-vessel coronary artery bypass surgery. Postoperatively, the patient has remained free of angina.

\section{DISCUSSION}

Coronary ostial stenoses are not uncommon after selective coronary perfusion for aortic valve replacement [1]. They usually develop in a short period of time $(<6$ mo) and can be bilateral [2].

From the Cardiology Division, Department of Internal Medicine, The University of Michigan School of Medicine, Ann Arbor, Michigan.

Received October 22, 1990; revision accepted December 4, 1990

Address reprint requests to Eric R. Bates, M.D., University Hospital B1 F245-0022, 1500 East Medical Center Dr., Ann Arbor, MI 481090022. 

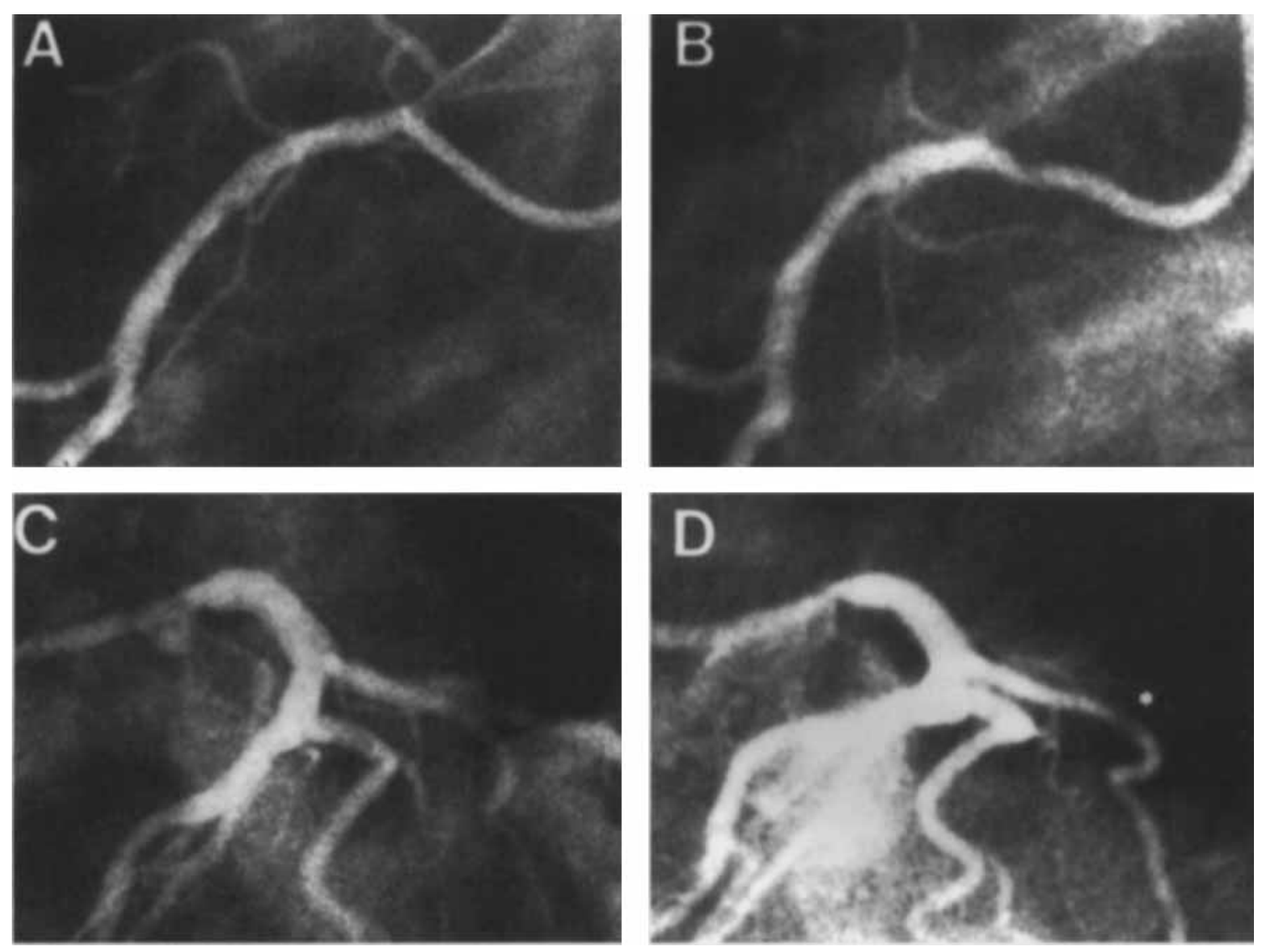

Fig. 1. Coronary arteriograms: Left anterior oblique projections of the right coronary artery at baseline (A) and 7 months later $(B)$ demonstrate a $60 \%$ ostial stenosis with lack of contrast reflux. Similarly, left anterior oblique cranial projections of the

left coronary artery at baseline (C) and 7 months later (D) demonstrate a $\mathbf{7 5} \%$ ostial stenosis. In addition, the left circumflex artery is occluded (asterisk).

Ostial stenoses following cardiac catheterization are much rarer. There have been several paired angiographic studies of the natural progression of coronary artery disease. In three of these studies with a total of 430 patients [3-5], only 2 cases of new left main coronary artery stenosis are mentioned. In one other study of $256 \mathrm{pa}$ tients [6], angiographic progression was studied by lesion location and percent diameter stenosis. Five patients developed progression at the origin of the right coronary artery: 4 with normal origins and 1 with $25-49 \%$ narrowing on initial study. No patient developed left ostial narrowing, and only 3 patients had left main stem progression.

One would expect a higher incidence of ostial stenoses following PTCA compared to cardiac catheterization because of added trauma from guide catheters and other hardware causing histologically documented endothelial injury [7]. Only 19 cases of left main coronary artery stenosis following PTCA have been reported [7-15]. Only 6 cases had normal appearing left main coronary arteries on initial angiograms; the remainder had luminal

irregularities or $30-40 \%$ stenoses. The site of subsequent narrowing was almost always the distal left main coronary artery and may have been related to balloon injury from dilating very proximal left anterior descending artery stenoses. The time to stenosis development ranged from 2 to 14 months and averaged 5-7 months.

The case presented here is unique because bilateral ostial stenoses developed 8 months after initial cardiac catheterization. In addition, the left circumflex artery occluded. Despite apparently aggressive coronary artery disease progression, the site of PTCA was widely patent on 5-month follow-up. In the previously reported cases in which the PTCA site at follow-up was mentioned, 3 of 10 cases restenosed. The factors responsible for the development of bilateral ostial stenoses in this patient are unknown. While endothelial injury to the ostia from catheter trauma is probably universal, subsequent ostial stenoses remains uncommon and unpredictable. When angina pectoris worsens following cardiac catheterization or PTCA, accelerated ostial stenosis should not be overlooked in the differential diagnosis. 


\section{REFERENCES}

1. Midell AI, DeBoer A, Bermudez G: Postperfusion coronary ostial stenosis; Incidence and significance. J Thorac Cardiovasc Surg 72:80-85, 1976.

2. Nakhjavan FK, Maranhao V, Goldberg H: Iatrogenic stenosis of the proximal portion of the coronary arteries. Am Heart J 83: 318-321, 1972.

3. Gensini GG, Kelly AE: Incidence and progression of coronary artery disease. Arch Intern Med 129:814-827, 1972.

4. Kramer JR, Matsuda Y, Mulligan JC, Aronow M, Proudfit WL: Progression of coronary atherosclerosis. Circulation 63:519-526, 1981.

5. Marchandise B, Bourassa MG, Chaitman BR, Lesperance J: Angiographic evaluation of the natural history of normal and mild coronary atherosclerosis. Am J Cardiol 41:216-220, 1978.

6. Bruschke AVG, Wijers TS, Kolsters W, Landmann J: The anatomic evolution of coronary artery disease demonstrated by coronary arteriography in 256 nonoperated patients. Circulation 63 : 527-536, 1981.

7. Waller BF, Pinkerton CA, Foster LN: Morphologial evidence of accelerated left main coronary artery stenosis: A late complication of percutaneous transluminal balloon angioplasty of the proximal left anterior descending coronary artery. J Am Coll Cardiol 9: 1019-1023, 1987.
8. Bashour TT, Hanna ES, Edgett J, Geiger J: Iatrogenic left main coronary artery stenosis following PTCA or valve replacement. Clin Cardiol 8:114-117, 1985.

9. Graf RH, Verani MS: Left main coronary artery stenosis: A possible complication of transluminal coronary angioplasty. Cathet Cardiovasc Diagn 10:163-166, 1984.

10. Hamad N, Pichard A, Oboler A, Lindsay J: Left main coronary artery stenosis as a late complication of percutaneous transluminal coronary angioplasty. Am J Cardiol 60:1183-1184, 1987.

11. Harper JM, Shah Y, Kern MJ, Vandormael MG: Progression of left main coronary artery stenosis following left anterior descending coronary artery angioplasty. Cathet Cardiovasc Diagn 13: $398-400,1987$.

12. Haraphongse M, Rossall RE: Subacute left main coronary stenosis following percutaneous transluminal coronary angioplasty. Cathet Cardiovasc Diagn 13:401-404, 1987.

13. Killen DA, Hamaker WR, Reed WA: Coronary artery bypass following percutaneous transluminal coronary angioplasty. Ann Thorac Surg 40:133-138, 1985.

14. Slack JD, Pinkerton CA: Subacute left main coronary stenosis: An unusual complication of percutaneous transluminal coronary angioplasty. Angiology 36:130-136, 1985.

15. Wayne VS, Harper RW, Pitt A: Left main coronary artery stenosis after transluminal coronary angioplasty. Am $\mathbf{J}$ Cardiol 61:459$460,1988$. 